\title{
Chain stiffness intensifies the reptation characteristics of polymer dynamics in the melt
}

\author{
Roland Faller* \\ Florian Müller-Plathe \\ Max-Planck Institut für Polymerforschung, Ackermannweg 10, 55128 Mainz, Germany
}

November 13, 2018

\begin{abstract}
The reptation concept in polymer dynamics is studied for model chains with added stiffness. The main idea of a chain diffusing inside a tube can be transferred from fully flexible chains although the renormalization onto a flexible chain of fewer Kuhn segments fails. The entanglement length shrinks with increasing persistence length. If entanglement length and persistence length come to the same order of magnitude the picture of a tight tube is better suited, in which chain segments can move only along the contour, any transverse motion being much reduced. Thus, as stiffness increases, the monomers loose their freedom to perform random walks inside the tube, the "Rouse-like" part of their dynamics.
\end{abstract}

Keywords: Molecular dynamics, Polymers

For the dynamics of polymer chains longer than the characteristic entanglement length in the melt the concept of reptation ${ }^{[1,2]}$ is widely accepted. In contrast to short chains which move isotropically and which can be described by the Rouse model, ${ }^{[3]}$ longer chains are constrained to an effective tube by topological entanglements due to the surrounding chains. The many chain problem may be replaced by an effective single chain problem with a mean field representation of the neighbors. This model has been developed for fully flexible polymers and is based on the Rouse model for local dynamics, which treats a polymer chain as a set of non-interacting beads connected by springs in a bath of homogeneous friction. The entanglement monomer number $N_{e}$ is the chain length at which the cross-over from Rouse to reptation dynamics occurs. ${ }^{[4]}$ As chain stiffness is added to the model, the Rouse description is no more appropriate for the local dynamics. ${ }^{[5]}$ So it becomes questionable if the standard reptation picture is still suitable for the dynamics of entangled stiff chains. After some earlier work on the reptation of almost rigid rods, ${ }^{[6]}$ recent theoretical work tries to adapt the reptation model to moderately stiff polymer chains. ${ }^{[7]}$

* present address: Department of Chemical Engineering, University of Wisconsin, Madison, WI 53706, USA 
The effective stiffness of a polymer is characterized by its persistence length $l_{p}$ which is defined as the exponential decay length of orientation correlations. This is the chain length for which a polymer keeps its orientation. Several theoretical models propose different scalings of the entanglement length with the persistence length stiffness. ${ }^{[6-11]}$ There is not even consensus whether the entanglement length rises or falls with stiffness. Scaling behaviors ranging from $N_{e} \propto l_{p}^{-3}$ to $N_{e} \propto l_{p}^{2}$ have been proposed.

The aim of this simulation study is to investigate the qualitative change of polymer dynamics from the standard Rouse/reptation picture as chain stiffness is introduced and increased. We show that quantities like the entanglement length or the tube diameter become somewhat diffuse and ambiguous in this case. Still, the concept of an entangled polymer moving in a tube holds; and irrespective of the way of defining the entanglement length, it can be shown to decrease with increasing persistence length $l_{p}$. Eventually, the two lengths become comparable and the chain dynamics changes to pure reptation dynamics without an underlying local short-time Rouse regime. One should note that most real polymers have some chain stiffness and they do show local dynamics very similar to our simulations as has been shown in recent NMR experiments. ${ }^{[12]}$ The interplay of entanglements and stiffness is sometimes discussed in terms of a packing length. ${ }^{[13,14]}$ The experimentally accessible region is, however, much smaller than the parameter area discussed in the following, i.e. in terms of stiffness experimental polymers are less different than they appear on first sight. Yet, simulations and experiments have shown that the Rouse model is not fully appropriate for real polymers especially at short ranges where local properties play an increasing role. ${ }^{[15-17]}$

We apply a well-described simulation model of polymer dynamics ${ }^{[4,18,19]}$ augmented with intrinsic stiffness ${ }^{[20,21]}$ along the polymer backbone. The chains consist of purely repulsive soft spheres connected by anharmonic springs and a harmonic bond angle potential. Constant temperature is maintained by Brownian dynamics. For details of the simulated systems see ref. 22. We have investigated systems containing up to 2000 chains of 2 to 1000 monomers of different stiffness ranging from fully flexible to a persistence length of five monomer diameters. The chains with no imposed stiffness (the original model ${ }^{[18]}$ ) have a persistence length of about one monomer diameter resulting from the excluded volume interaction. The units used in this article are Lennard-Jones reduced units with monomer mass $m$, monomer diameter $\sigma$ and interaction strength $\epsilon$ set to 1 . For the exact definition and the potentials see ref. 21 . As melts with persistence lengths much larger than five turn nematic, ${ }^{[23]}$ they were not investigated. The time-step was set to 0.01 time units, the density to $\rho=0.85 \sigma^{-3}$ and the average bond length turns out to be $0.97 \sigma$.

Mean-squared displacements (MSD) of central monomers in a chain, in the following referred to as $g_{1}(t)$, are a key property both in theory and simulation, as they illustrate the different dynamic regimes of reptation. The dependence of $g_{1}$ on time scales according to theory with four dynamical exponents in the reptation case: ${ }^{[2]}$ at small times the monomers behave as in the Rouse model $\left(g_{1} \propto t^{1 / 2}\right)$; after the so-called entanglement time $\tau_{e}$, the motion is constrained to a tube, thus the Rouse relaxation is one-dimensional along a random walk leading by transformation to three dimensions 

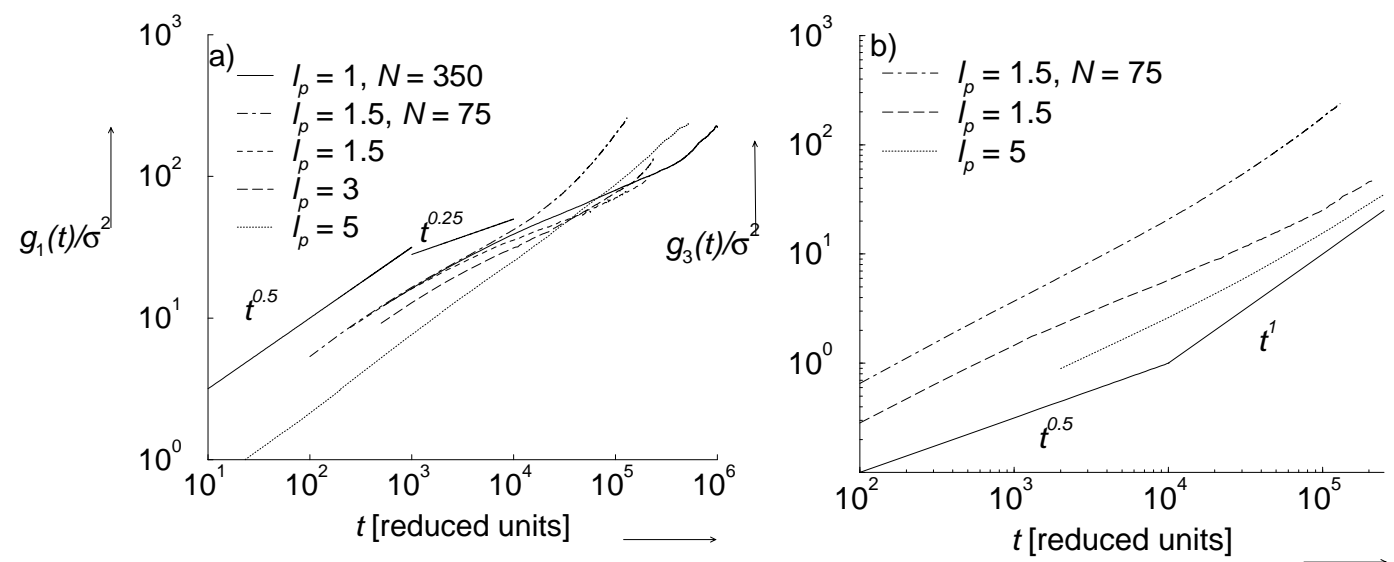

Figure 1: Mean squared displacements of a) monomers in the central part of the chain b) center of mass. Chains of length 200, unless stated otherwise.

to $g_{1} \propto t^{1 / 4}$; at the Rouse time $\tau_{R}$ the internal degrees of freedom are relaxed but the motion is still confined to the tube $\left(g_{1} \propto t^{1 / 2}\right)$; and after the chain leaves the tube at the disengagement time $\tau_{d}$ free diffusion $\left(g_{1}(t) \propto t\right)$ is observed. This behavior was confirmed by simulations of fully flexible chains. ${ }^{[4,19,24]}$ The first three regimes can be seen for the $l_{p}=1$ curve in Figure 1 a.

Depending on stiffness we find two different classes of dynamics. Up to a persistence length of $l_{p} \leq 3$, the chains behave qualitatively like flexible chains. Chains of length $N=200$ are at the density used clearly entangled for $l_{p}=1.5$ and $l_{p}=3$. Even the chains of length 75 and persistence length of only 1.5 exhibit a slowdown to $g_{1}(t) \propto t^{0.3}$ (Figure 1a), which is more pronounced than for fully flexible chains at $N=200 .{ }^{[24]}$ With increasing persistence length the value of the mean-squared-displacement value at the crossover $\tau_{e}$ from $t^{1 / 2}$ to $t^{1 / 4}$, which is associated with the square of the tube diameter $d_{T}$ shrinks slightly. This is a first indication that the entanglement length $\left(\approx d_{T}^{2} / l_{p}\right.$ for flexible chains) decreases with increasing persistence length. The time $\tau_{e}$ can be transformed into the relaxation time of a chain, from which the entanglement length (i.e. the number of monomers) $N_{e}$ can be obtained. ${ }^{[2,4]}$ The Rouse model is standardly employed in this mapping procedure. As - in the presence of stiffness - the Rouse dynamics is no longer appropriate even for short chains, $N_{e}$ becomes somewhat ambiguous.

For the system with persistence length $l_{p}=5$ not even the standard reptation scenario is recovered, as the $t^{1 / 4}$ regime is not observed. In the flexible case the tube diameter $d_{T}$ estimated from the crossover in the MSD to $t^{1 / 4}$ is about $\sqrt{40} \sigma \approx 6 \sigma$, and in Figure 1a it can be seen that it decreases further with $l_{p}$. Hence, it can be safely assumed that, for the $l_{p}=5$ chains, the tube diameter is equal to or smaller than the persistence length. As a result, stiff chains cannot wiggle freely in their tube but have to follow closely the primitive path. This is in marked contrast to the original Rouse/reptation model, in which chains perform a random walk inside their 
tubes. Now, the chain can only perform reptation moves because there is no transverse freedom to explore. On local scales, the stiffness hinders the transverse motion and, on longer scales, the entanglements prevent it. The tube itself appears worm-like rather than like a random walk. ${ }^{[7]}$ It does not differ much from the chain whose worm-like nature is e.g. visible in its static structure factors. ${ }^{[21]}$ Still, the limit of persistence length large compared to entanglement length is not approached.

Note that the different MSDs intersect in Figure 1a. The stiffer chains are faster in an intermediate regime. For long times (not shown), however, we expect that the size of the ellipsoid spanned by the chain defines the ultimate diffusion speed. Thus, the flexible chains should be faster again. This is indicated e.g. by comparing the chains with $l_{p}=3$ and $l_{p}=5$, where a second intersection is very probable for longer times (Figure 1a). A simple argument assuming strictly reptating chains compared to the standard reptation supports this picture: The final diffusion depends only on the extension, so that the stiffer chains are slower. However, for these chains the first two regimes are missing due to the lack of the Rouse regime. This was confirmed by an analysis of the Rouse modes. ${ }^{[25]}$ They start directly with the third regime so that the slowdown to $t^{1 / 4}$ for the flexible chains lets the stiffer chains overtake. ${ }^{[25]}$

The centers of mass of chains can diffuse "freely" in the unentangled case $\left(N<N_{e}\right)$, whereas in the entangled case a sub-diffusive motion arising from the constraining to the curvilinear tube is found up to the Rouse time $\tau_{R}$ (Figure 1b). The chains with $l_{p}=5$ and $N=200$ are highly entangled, which is not only visible by the subdiffusive nature of the MSD of the center of mass $g_{3}(t)$ but also by their reorientation behavior. ${ }^{[22,25]}$

According to the Rouse model, the center of mass diffusion coefficient $D$ for $t \rightarrow \infty$ scales with $N^{-1}$. Deviations from this in the long chain regime are normally attributed to entanglements. ${ }^{[4]}$ Figure 2 shows the dependence of $D N$ on the chain length for different stiffnesses. The fully flexible model $l_{p}=1$ shows Rouse behavior up to $N \approx 32$ and then the diffusion coefficient falls off with $D \propto N^{-2}$. This picture does not directly extend to higher persistence lengths for which there are again two classes of dynamics. For persistence length $1.5 \leq l_{p} \leq 3$ there is a first plateau (Rouse) at very short chain lengths $(N \lesssim 10)$. Then the diffusion constant decreases faster than $N^{-1}$. A second short plateau is found $(10 \lesssim N \lesssim 20)$ followed by a decay as the entanglement length is approached. For higher stiffness $\left(l_{p}=5\right)$ no plateau at all is found, indicating that the Rouse model cannot be applied even for the shortest chains. For this system, a Rouse scaling of the autocorrelation functions of the Rouse modes is not possible either. ${ }^{[25]}$ The Rouse regime is "squeezed out" between the short local modes and the long modes hindered by entanglement. With increasing $l_{p}$ longer wavelengths are influenced by stiffness. As the entanglement length decreases at the same time shorter wavelengths become entangled. This eventually annihilates the regime of random (Rouse) motion.

By rescaling the abscissa to match the diffusion coefficient at $N_{e}=32$ (Figure 2b) one can derive estimates of entanglement lengths: $N_{e}\left(l_{p}=1.4\right)=15, N_{e}(3)=8$, and $N_{e}(5)=6$. For flexible chains the entanglement length coincides with the value derived by the crossover in the $g_{1}$ function. ${ }^{[4,19]}$

The effective entanglement lengths are not as sharply defined as in the flexible case. Even for fully flexible chains there are several definitions of the entanglement length, ${ }^{[19]}$ 

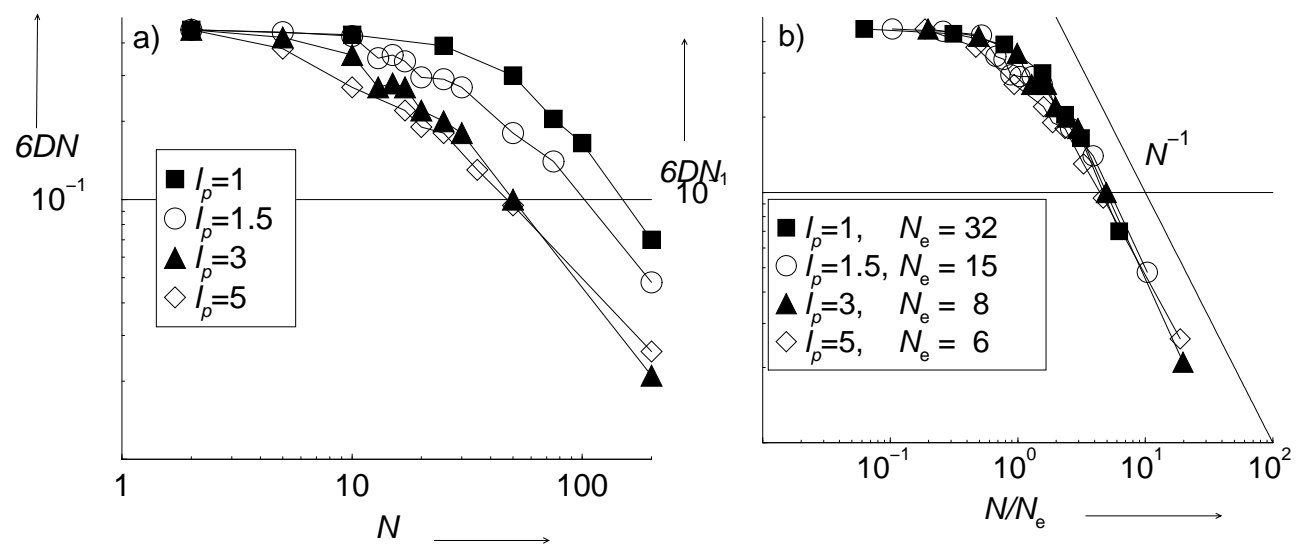

Figure 2: a) Center of mass diffusion constant for different chain lengths in Rouse scaling, b) same as a) but rescaled empirically to define a entanglement length. Below $N=50$ the errors are smaller than the symbol sizes. In both figures the filled squares correspond to $l_{p}=1$, the open circle to $l_{p}=1.5$, the filled triangle to $l_{p}=3$ and the open diamond to $l_{p}=5$. The entanglement lengths depending on persistence length used in part b) are $N_{e}(1)=32, N_{e}(1.5)=15, N_{e}(3)=8$, and $N_{e}(5)=6$.

and this ambiguity becomes worse for moderately stiff chains which have two plateaus in $D N(N)$. However, the decrease of $N_{e}$ with $l_{p}$ observed is systematic, independent of the details how $N_{e}$ is actually extracted. Moreover, the scaling described above leads to a collapse onto a master curve to a reasonable approximation (Figure 2b).

The previous section showed that stiffness in connection with topological entanglements leads to a novel dynamics of chains in the melt. The character of the local motions can be investigated in more detail. The correlation function

$$
\Pi_{d}(t)=\left\langle P_{2}\left(\frac{\vec{x}_{i}(t)-\vec{x}_{i}(0)}{\left|\vec{x}_{i}(t)-\vec{x}_{i}(0)\right|} \cdot \frac{\vec{x}_{i+d}\left(\frac{t}{2}\right)-\vec{x}_{i-d}\left(\frac{t}{2}\right)}{\left|\vec{x}_{i+d}\left(\frac{t}{2}\right)-\vec{x}_{i-d}\left(\frac{t}{2}\right)\right|}\right)\right\rangle
$$

connects the direction of the displacement of a monomer with the local chain direction, where $P_{2}$ is the second Legendre polynomial. The length of the corresponding chain segment is equal to $2 d$. For $d=1$, it is defined by the two nearest neighbors. This function indicates if the beads move predominantly in the chain direction or if their motion is not correlated to this direction. As the highly entangled $l_{p}=5$ systems have fewer transversal degrees of freedom, the correlation is high, signaling a dominance of reptation (Figure 3a). There is evidence for a finite correlation length as the maximum correlation is not encountered for $d=1$ at $l_{p}=5$ (Figure 3a). For $d=10(\approx \mathrm{Kuhn}$ segment length) the correlation is larger than for $d=1$ and for $d=50$. At local scales some transverse degrees of freedom in the tube remain and on the really large scale the static structure shines through. As the statics is not influenced by entanglements, it can be described by a random walk of blobs. ${ }^{[21]}$ Figure $3 \mathrm{~b}$ provides yet more evidence 

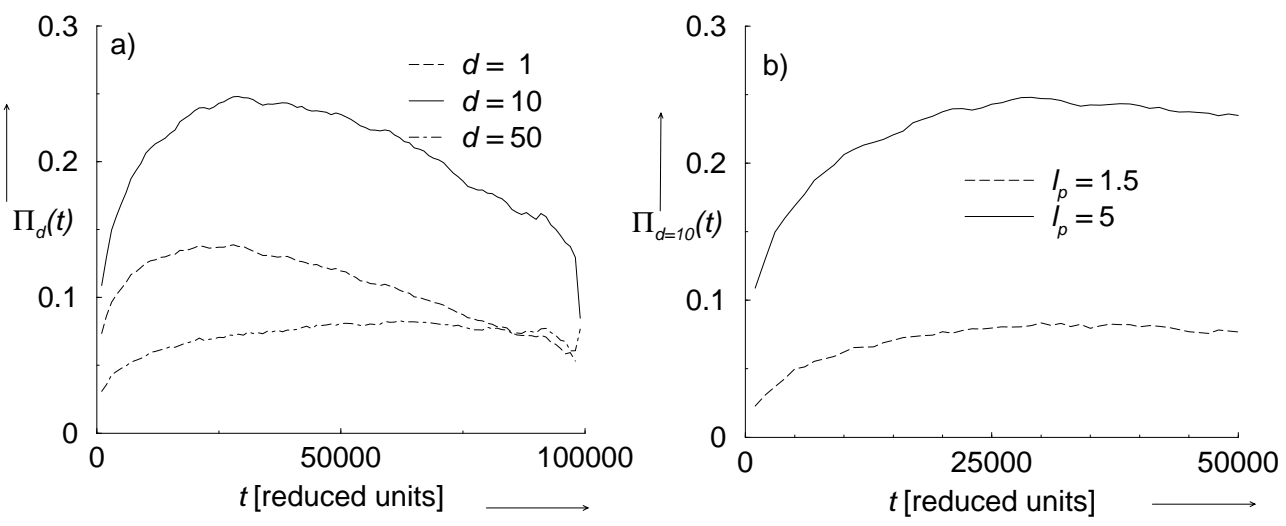

Figure 3: Correlation of inner chain direction vectors with their displacements $\Pi_{d}$ a) Different lengths of segments for $l_{p}=5, N=200$. b) Comparison of different stiffnesses $N=200, d=10$.

for the enhancement of reptation by added stiffness: At all times the correlation of motion with chain direction is stronger for $l_{p}=5$ than for $l_{p}=1.5$.

The tube is visualized in Figure 4. The numbers denote the positions of the chain end in the order they appear. The end clearly drags back into the tube and moves out again into a new tube. Note that the figure includes all monomers without any preaveraging. One can distinguish stretched segments of the tube in which the chain is also stretched and shows almost no crumpling over the entire simulation. These rigid segments can undergo a subdiffusive translation while keeping their orientation. Simultaneously reptation in the tube is possible. This illustrates the concept of "chain reptation without Rouse motion" for stiff polymers. This expresses itself also in the fact that reorientation correlation functions of bond-vectors decay algebraically on short time scales. And monomers propagate through space without losing all of their correlation. ${ }^{[22]}$

From the dynamic structure factor the tube diameter can be inferred. For Rouse dynamics, the structure factor decays uniformly, whereas reptation leads to the development of a plateau. Recent experiments showed the presence of reptation e.g. in polyethylene melts. ${ }^{[26]}$

The dynamic structure factor of the flexible chains can be well fitted by the formula of Kremer and Binder. ${ }^{[27]}$

$$
\begin{aligned}
\frac{S(k, t)}{S(k, 0)}= & \left\{1-\left(\frac{k d_{T}}{6}\right)^{2} f\left[k^{2} l_{b}^{2}\left(\frac{3 k_{B} T}{l_{b}^{2} \zeta} t\right)^{1 / 2}\right]\right\} \times \\
& \frac{8}{\pi^{2}} \sum_{p=1}^{\infty} \frac{1}{(2 p-1)^{2}} \exp \left(-\frac{t(2 p-1)^{2}}{\tau_{d}}\right) \\
f(u)= & \exp \left(\frac{u^{2}}{36}\right) \operatorname{erfc}\left(\frac{u}{6}\right) .
\end{aligned}
$$




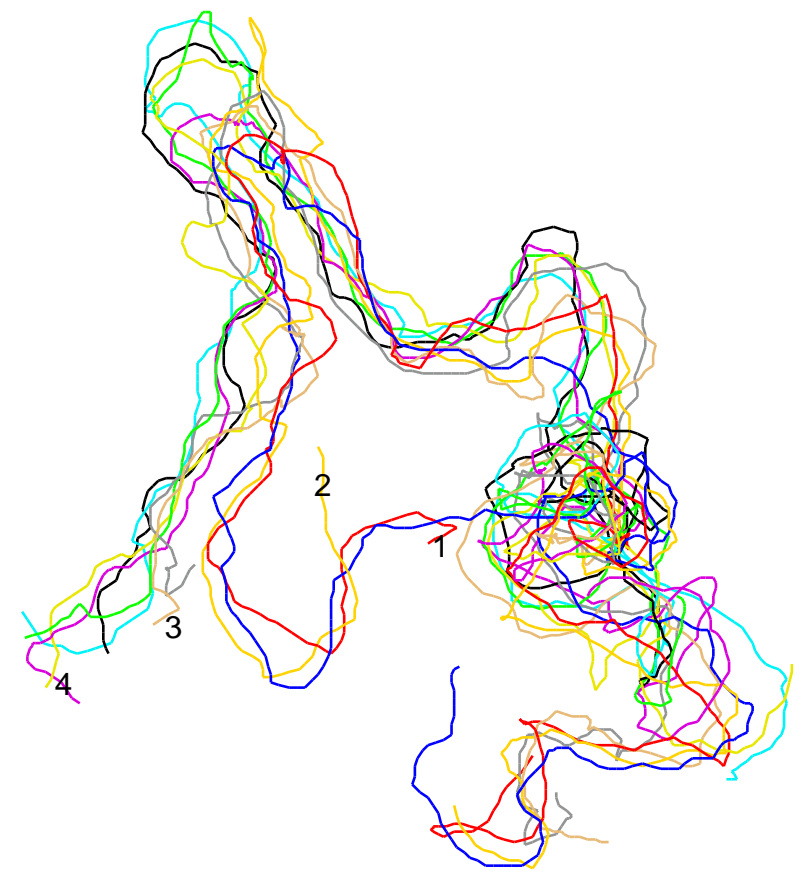

Figure 4: One chain in the system with $l_{p}=5$ and $N=200$. The different frames are at a time distance of $\Delta t=10000$. 

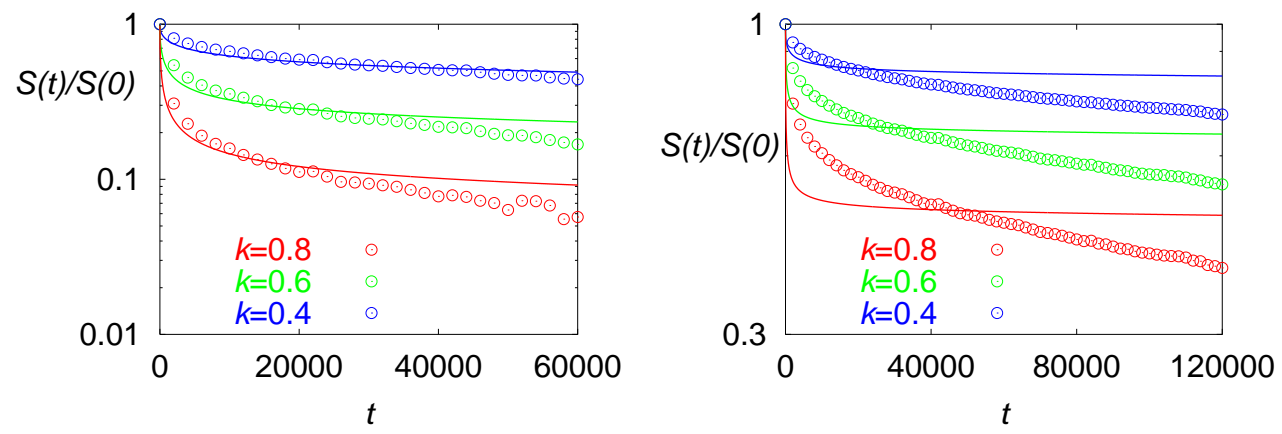

Figure 5: Normalized dynamic structure factors for $N=200$ and $k=0.4,0.6$, and 0.8 . for a) $l_{p}=1.5$ and b) $l_{p}=5$. Note the different scales on the axes.

The only fit parameter employed is the tube diameter $d_{T}$, the Rouse friction $\xi$ was obtained from the decay of the Rouse modes, the disengagement time $\tau_{d}$ can only be estimated assuming the standard picture. Still, the fit works reasonably well for $l_{p}=1.5$ (Figure 5a), resulting in a tube diameter of 12.2 for chains of length 200 which is most probably too high compared to the value obtained from the monomer displacements (see above). For flexible chains it was shown that this discrepancy vanishes only in the limit of very large $N .{ }^{[19]}$ For the even shorter chains of length 75 , we derive a value of 15.4 , which indicates a tube diameter decreasing with length. It also decreases with stiffness; for $l_{p}=5$ Equation( 1) yields 6.1. However, the fit formula is not really appropriate to the stiff chains as the local Rouse dynamics on which it is based is absent (Figure 5b). Thus, this shows again that these chains are subject to a dynamics which cannot be described in terms of standard reptation theory. Still, we used the formula to yield at least a (rather crude) estimate of the tube diameter. In spite of this uncertainty the difference is large enough to conclude that also in this case the length scale governing the motion pattern, here $d_{T}$, decreases strongly with increasing stiffness.

Our simulations show that the lengths commonly associated with a change in chain diffusion mechanism, the entanglement length $N_{e}$ and the tube diameter $d_{T}$, decrease as the intrinsic stiffness of the chains increases. As a result, the motion patterns change qualitatively already for small stiffness (persistence length $l_{p}=1.5-3$ monomer diameters) and become completely different from that of flexible chains when $N_{e}$ and/or $d_{T}$ become comparable to or even smaller than $l_{p}$ (as is the case for $l_{p}=5$ ). For $l_{p}=5$, the cooperation of entanglements and stiffness obstructs isotropic random walk motion on all scales, the Rouse regime disappears and the chain reptates along its narrow tube from the beginning. The absence of Rouse dynamics distinguishes this situation from the standard de Gennes - Doi - Edwards picture. ${ }^{[1,2]}$ We suggest the term strong reptation for this scenario. A similar transition has been proposed by Morse ${ }^{[7]}$ who distinguishes between loose and tight entanglement. One should note that most real polymers do have persistence lengths larger than one monomer diameter. Even 
something commonly considered flexible like polyisoprene has $l_{p}=1.5-2 .{ }^{[25,28]}$ Our results suggest that even to such polymers the standard Rouse/reptation model can only be applied with caution. We have found deviations from the predictions of the standard model also for the reorientation of local chain segments, ${ }^{[22]}$ which are in good agreement with NMR measurements on polybutadiene melts. ${ }^{[12]}$

None of the existing theoretical models can completely explain the observed behavior. In the low persistence length region the packing length concept ${ }^{[11]}$ gives a useful power law dependence $N_{e} \propto l_{p}^{-2}$ but in the region where the two lengths become comparable there is not yet an analytic understanding.

\section{Acknowledgements}

We thank B. Dünweg, R. Everaers, A. Heuer, K. Kremer, and M. Pütz for fruitful discussions. Financial support from the German ministry of research (BMBF) is gratefully acknowledged.

\section{References}

[1] P.-G. de Gennes, J. Chem. Phys. 1971, 55, 572-579.

[2] M. Doi, S. F. Edwards, The Theory of Polymer Dynamics, vol. 73 of International Series of Monographs on Physics, Clarendon Press, Oxford, 1986.

[3] P. E. Rouse, 1953, J. Chem. Phys. 1953, 21, 1272-1280.

[4] K. Kremer, G. S. Grest, J. Chem. Phys. 1990, 92, 5057-5086.

[5] L. Harnau, R. G. Winkler, P. Reineker, J. Chem. Phys. 1997, 106, 2469-2476.

[6] T. Odijk, Macromolecules 1983, 16, 1340-1344.

[7] D. C. Morse, Macromolecules 1998, 31, 7030-7043.

[8] M. Doi, J. of Polymer Science: Polymer Symposium 1985, 73, 93-103.

[9] S. Wu, J. of Polymer Sci.: Part B Polymer Physics 1989, 27, 723-741.

[10] T. Sato, A. Teramoto, Macromolecules 1991, 24, 193-196.

[11] L. J. Fetters, D. J. Lohse, D. Richter, T. A. Witten, A. Zirkel, Macromolecules 1994, 27, 4639-4646.

[12] R. Graf, A. Heuer, H. Spiess, Phys. Rev. Letters 1998, 80, 5738-5741.

[13] L. J. Fetters, D. J. Lohse, W. W. Graessley, J. of Polymer Science Part B: Polymer Physics 1999, 37, 1023-1033. 
[14] L. J. Fetters, D. J. Lohse, S. T. Milner, W. W. Graessley, Macromolecules 1999, 32, 6847-6851.

[15] D. Richter, L. Willner, A. Zirkel, B. Farago, L. J. Fetters, J. S. Huang, Macromolecules 1994, 27, 7437-7446.

[16] W. Paul, G. D. Smith, D. Y. Yoon, Macromolecules 1997, 30, 7772-7780.

[17] V. A. Harmandaris, V. G. Mavrantzas, D. N. Theodorou, Macromolecules 1998, 31, 7934-7943.

[18] G. S. Grest, K. Kremer, Phys. Rev. A 1986, 33, R3628-R3631.

[19] M. Pütz, K. Kremer, G. S. Grest, Europhysics letters 2000, 49, 735-741.

[20] U. Micka, K. Kremer, Europhysics letters 1997, 38, 279-284.

[21] R. Faller, A. Kolb, F. Müller-Plathe, Phys. Chem. Chem. Phys. 1999, 1, 20712076.

[22] R. Faller, F. Müller-Plathe, A. Heuer, Macromolecules 2000, 33, 6602-6610.

[23] A. Kolb, PhD thesis, MPI für Polymerforschung and Universität Mainz 1999.

[24] B. Dünweg, G. S. Grest, K. Kremer, in Numerical Methods for Polymeric Systems (Ed. S. G. Whittington), Springer, 1998, Vol. 102 of IMA Volumes in Mathematics and its Applications, pp. 159-196.

[25] R. Faller, PhD thesis, MPI für Polymerforschung and Universität Mainz, 2000, published at http://archimed.uni-mainz.de/pub/2000/0063.

[26] P. Schleger, B. Farago, C. Lartigue, A. Kollmar, D. Richter, 1998, Phys. Rev. Letters 1998, 81, 124-127.

[27] K. Kremer, K. Binder, J. Chem. Phys. 1984, 81, 6381-6394.

[28] R. Faller, F. Müller-Plathe, D. Theodorou, M. Doxastakis, Macromolecules in press 2001. 